\title{
Query Processing Methods for Connectivity Search in Visual Databases Using $R^{+}$-tree
}

\author{
Myeong-A Kang and Ki-Joune Li \\ Department of Computer Science \\ Pusan National University \\ Kumjeong-Ku, Pusan, Korea 609-735 \\ e-mail : \{makang,lik\}@spatios.cs.pusan.ac.kr \\ fax: +82515102208
}

\begin{abstract}
Recently, spatial database systems have been developed and widely used in many applications, including visual database systems. However, the efficient query processing in visual database systems becomes a crucial problem, due to their large volume of data. It is furthermore difficult to reuse conventional query processing techniques for visual database systems, since types of queries used in visual databases differs from the existing ones in conventional database systems. New query processing methods are therefore required. In visual database systems, it is important to represent semantic information which include topological relationship of visual objects. One of the types of queries most frequently used to represent topological relationship in visual database systems is connectivity search. In this paper, we propose several spatial query processing methods for connectivity, using $\mathrm{R}^{+}$-tree as the underlying indexing method without any topological data. We introduce the concept of $\theta$-partial $R^{+}$-tree and its construction algorithm, which describes any kind of spatial query processing procedure depending on the specification of $\theta$-condition. Two query processing methods for connectivity are derived from $\theta$-partial $\mathbf{R}^{+}$-tree construction. We analyze the performance of our methods by simulation. It proves that our methods for connectivity search significantly reduce the number of disk accesses as well as CPU time.
\end{abstract}




\section{INTRODUCTION}

Spatial database systems have been developed and widely used in many applications, including visual database systems [1]. However, due to their large volume of data, the efficient query processing in spatial database systems becomes a crucial problem. And since types of queries used in spatial databases differs from the existing ones in conventional database systems [13], it is difficult to reuse conventional query processing techniques for spatial database systems. New query processing methods are therefore required.

The types of spatial queries demanded in these applications are diverse. They can be roughly divided into two categories: geometric queries and topological queries. Until now, only a small set of queries has been rigorously studied for their processing [2]. Moreover, most of the proposed spatial query processing techniques are for only geometric purposes, such like distance, intersection or containment [14]. These methods are generally based on position data of spatial objects. In addition to geometry, topological information - for example, the shortest path between two given spatial objects- plays an important role in spatial databases. But only a few researches have been done for topological query processing [2][3][4].

Among the topological queries, the query about connectivity is the most basic, but complex and expensive one [1]. It is a kind of spatial query which asks to find out the paths between two given spatial objects. For example, one may ask to visualize all cities reachable from a given city via railroads. In this case, the connectivities between the given city and others are to be verified. Another example of query about connectivity is the shortest path query between two objects. In order to process such a query, we first have to find out all paths between the objects, that is, the connectivity of two objects. In a similar way, processing query about connectivity, therefore constitutes a major part of spatial or visual database systems.

In general, processing query for connectivity is very complex, expensive and difficult to implement. The major difficulty lies in disk access time. Since query processing for connectivity requires a great number of disk accesses, it spends a considerable amount of response time. In this paper, we propose several query processing methods for connectivity, focusing our efforts on the reduction of disk access frequency. We use filtering and refinement procedure for the query processing, which significantly remove unnecessary disk accesses. Compared with other approaches [7][8], no additional information is needed for the query processing, except $\mathrm{R}^{+}$-tree as the indexing tree of objects. It allows us to maintain database more dynamically. A generalized concept of filtering $\mathrm{R}^{+}$-tree, $\theta$-partial $\mathrm{R}^{+}$-tree, is introduced in this paper to formalize and extend our methods.

The organization of this paper is as follows. In Section 2, an overall procedure of query for connectivity is presented. We introduce the concept of $\theta$-partial $\mathbf{R}^{+}$-tree that can be built from $\mathrm{R}^{+}$-tree for efficient query processing in section 3 . We also show how $\theta$-partial $\mathbf{R}^{+}$-tree can be used generally and adaptively in spatial query processing. In Section 4, we apply $\theta$-partial tree to query processing for connectivity. We propose two methods; a simple one and an improved one. In Section 5, we present results of our experiments to compare the performance of the methods. And we conclude our paper in section 6. 


\section{QUERY PROCESSING FOR CONNECTIVITY}

When analyzing visual information, we often need topological relationship between visual objects in database. Connectivity between two objects is one of the most frequently inquired relationship. For example, as shows Figure 1, suppose that we want to know if the buildings in area $A$ be supplied with electricity, when transform substation $T$ is crashed. In order to answer to this question, we must find out possible paths from power plant $P$ to $A$ via power distribution network, that is, the connectivity of distribution network between power plant and its consumers in area $A$. If there exist connected paths, we can find an alternative among them, such like, the shortest path.

The difficulties lie in the size of data volume. In order to process query for connectivity, a great number of disk accesses and a large amount of computation may be often needed due to its large volume of data. Figure 2 illustrates an example of such a case.

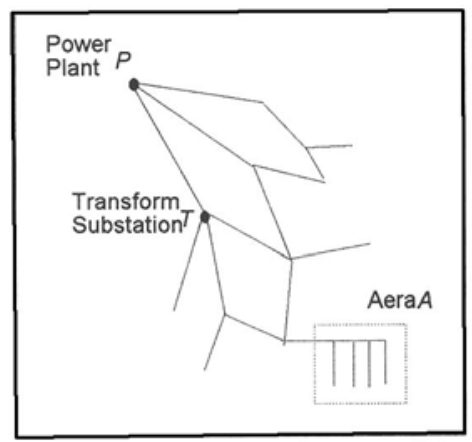

Figure 1 Example of Connectivity.

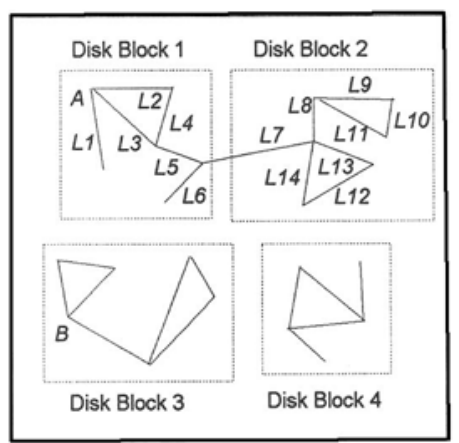

Figure 2 Storing Segments on Disk.

In Figure 2, in order to find the connected path between $A$ and $B$, we must scan at least three disk blocks containing line segments which are directly or indirectly connected with $A$ or $B$. That is, in order to find line segments $\{L 1, L 2, L 3\}$, which are directly connected with $\mathrm{A}$, we read disk block 1 . On disk block 1 , we can also find out line segments $\{L 4, L 5\}$ connected $\{L 1, L 2, L 3\}$, i.e.. But to find out line segments $\{L 6, L 7\}$, which are connected with $\{L 4, L 5\}$, we must read disk block 2 in turn. In a similar way, we read block 3 . As the number of line segments grows, the number of disk accesses required may also increase in a considerable way. In an extreme case, we must scan all disk blocks to find connected paths.

Another problem we meet in query processing is that we can not guaranty that all neighbor line segments be stored in the same disk block. For example, if we store line segments on disk blocks by the order of insertion, $L 2$ and $L 3$ are no longer in the same block. Furthermore, we do not know where $L 3$ is stored. It means that we must scan all blocks to find line segments directly connected with $A$. In such a case, the number of disk accesses can exceeds the number of disk blocks actually used.

Very little attention has been paid on the query processing methods for connectivity except some recent researches such as [3]. The simplest method to find connectivity between two objects may be graph traversal algorithms or Dijkstra's single-source all-pairs algorithm[5]. But 
it has a number of serious problems. The most serious problem among them is that this method do not takes into account $\mathrm{I} / \mathrm{O}$ costs. Even though the complexity by depth-first search is $O(e)$, it merely explains CPU time, and our concern is rather $\mathrm{I} / \mathrm{O}$ cost than CPU time.

Another possible approach is transitive closure algorithm [6]. By computing transitive closure of visual objects, we can easily find connectivity of two objects. But this approach has an important drawback, that computing and maintaining transitive closure cost too much CPU and $\mathrm{I} / \mathrm{O}$ time. For example, when a visual object is inserted, the transitive closure of the objects must be recomputed, which makes this method far from being dynamic.

Several graph traversal algorithms were proposed by [3] to find connectivity or shortest path on a massive graph, which is too large to fit in main memory and must be stored on disk. They use branch and bounding searching algorithms to prune the search space. These methods seem very attractive, since we may apply them to the query processing for connectivity. But even though this method may reduce $\mathrm{I} / \mathrm{O}$ cost, their $\mathrm{I} / \mathrm{O}$ cost is still unknown, and in worst case where the heuristics do not perform well, the number of disk accesses could increase by considerable amount.

The methods mentioned above are primarily based on only topological property. They represent the relationship between objects as a graph, and by using their topological properties, they try to find connectivity between objects. But by exploiting geometric information as well as topological one, we can significantly improve the performance of method. This is the basic concept of our methods.

In section 3 and 4 , we present how connectivity search query can be efficiently performed, by using only geometric information. First, our approach does not require any additional topological information as above methods, and uses only geometric information and spatial access method such as $\mathrm{R}^{+}$-tree which is a primitive requirement in any kind of visual database systems [9][10][11]. Therefore, storage can be reduced and information redundancy of object can be prevented. Second, the methods presented in this paper are dynamic at frequent insertion or deletion. Third, we significantly reduce $I / O$ time by using $\theta$-partial $R^{+}$-tree which will be proposed in this paper. It can be built from $\mathrm{R}^{+}$-tree and is a formal notation of filtering and refinement spatial query processing methods. This notation helps us to describe and implement spatial query processing with ease, which are, in general complicated and diverse in visual database.

\section{$3 \theta$-PARTIAL $\mathrm{R}^{+}-\mathrm{TREE}$}

The simplest method to find connectivity between objects in visual databases is to load all visual objects in main memory and process without any disk access. But it is practically impossible to keep all objects in main memory due to the large volume of visual data, and it is, thus inevitable to store visual objects in disk storage. The goal of query optimization for connectivity should be rather to reduce disk I/O frequency.

Our approach is to extend and generalize the spatial join query processing method appeared in [9]. According to this method, spatial query processing uses $\mathrm{R}^{+}$-tree for its spatial access method and is divided into two steps; filtering step and refinement step. Each step of this method is explained below; 
i. Filtering step : find all objects whose $\mathrm{MBR}^{*}$ satisfy the query condition.

ii. Refinement step : for these objects, check if they really fulfill the query condition.

The performance of query processing is primarily determined by filtering. The more objects are filtered out, the better is the performance. But the filtering in query processing for connectivity search is very complicated, and we need its formal notation. In this section, we try to formalize filtering process by $\theta$-partial tree.

\section{Definition 1 : Partial Tree}

Suppose that $T$ is a tree and $R$ is its root node. $T_{P}$ is a partial tree of $T$ if and only if

1. the root node of $T_{P}, R_{P}$ is identical to $R$;

2. when $C(R)$ is the set of child nodes of $R, C\left(R_{P}\right) \subseteq C(R)$;

3. each subtree of $T_{P}$ is a partial tree of a subtree of $T$;

An example of partial tree is illustrated by Figure 3. A partial tree $T_{P}$ is derived from $T$ such that their root nodes are identical and internal nodes on each level in $T_{P}$ is a subset of $T$ on corresponding level. We now define $\theta$-partial tree which is a key concept of our paper.

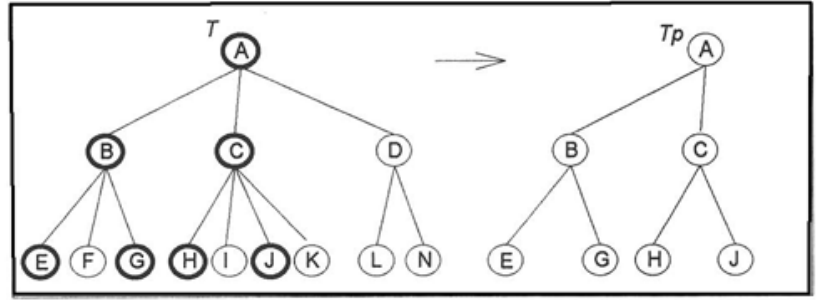

Figure 3 Example of Partial Tree.

Definition 2. $\theta$-Partial Tree and $\theta$-Partial $R^{+}$-Tree

Let $T$ be a tree. Then $T_{\theta}$ is a $\theta$-partial tree if and only if

1. $T_{\theta}$ is a partial tree of $T$;

2. on each level of $T_{\theta}$, the set of sibling nodes satisfies condition $\theta$;

And when $T$ is a $\mathrm{R}^{+}$-tree, we call its $\theta$-partial tree as $\theta$-partial $\mathrm{R}^{+}$-tree.

In order to construct a $\theta$-partial tree from a given tree, we must examine if selected nodes on each level satisfy the condition given by $\theta$. Figure 4 shows an example of $\theta$-partial tree where $\theta$ is given as follows ;

\footnotetext{
* Two terminologies, MBR(Mininal Bounding Rectangle) and node in $\mathrm{R}^{+}$-tree are interchangeable in this paper for the reason of convenience, even though their meanings are slightly different.
} 
$\theta: N=\left\{n \mid n \in \operatorname{sibling}(T, i), \sum_{n \in N} n \cdot d a t a \leq 20\right\}$,

where sibling $(T, i)$ is the set of all sibling nodes of $T$ on level $i$.

It should be noted that when building a $\theta$-partial tree, selection of sibling nodes on each level is not affected by nodes on upper levels, except that their parents have been already selected.

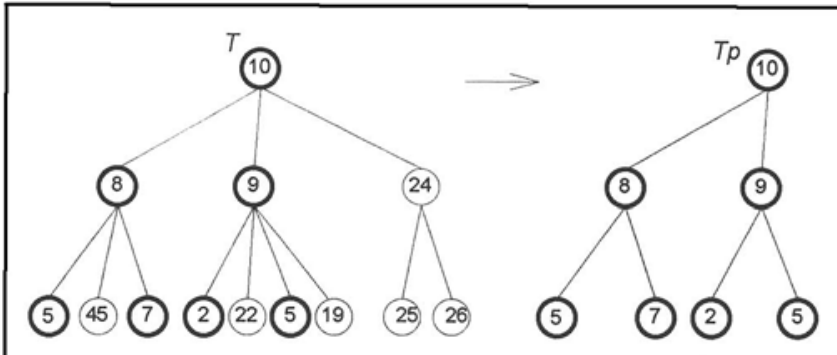

Figure 4 Example of $\theta$-partial tree.

Now, we see how $\theta$-partial tree can be used for spatial query processing. Suppose that a set of visual objects and its $\mathrm{R}^{+}$-tree $T$ are given as Figure 5(a) and we specify condition $\theta$ as

$\theta: N(i)=\{n \mid n$ is a node on level $i$ and $\operatorname{MBR}(n)$ intersect with region $W\}$

It means that we want to find visual object which intersects with a given searching area $W$. Then we can derive a $\theta$-partial tree from $T$ as shown by Figure 5(b).

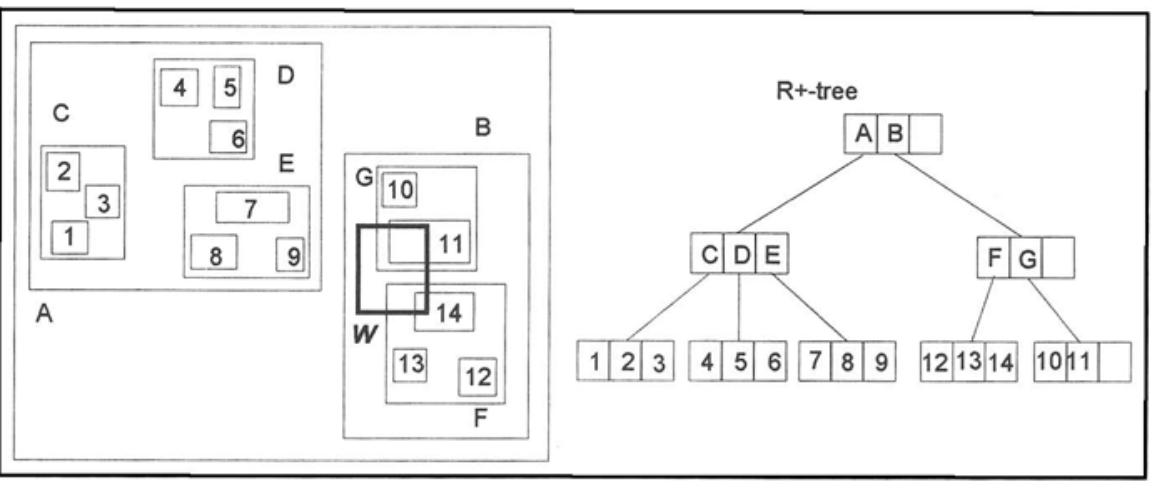

Figure 5 (a) $\mathrm{R}^{+}$-tree of a set of visual objects. 


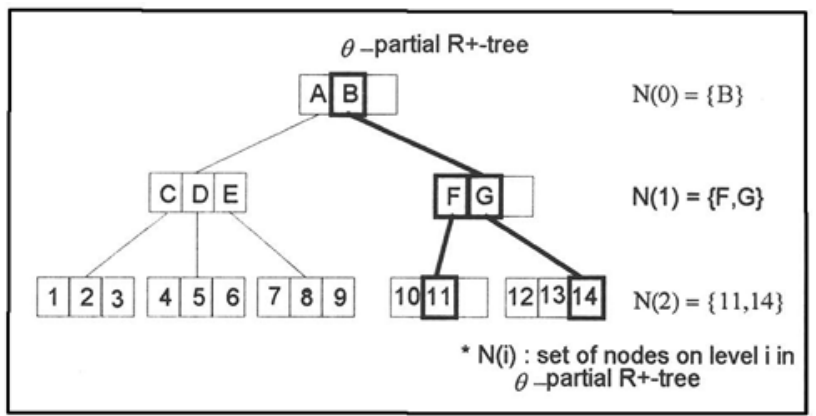

Figure 5(b) $\theta$-partial $\mathrm{R}^{+}$-tree.

In Figure 5(b), we find that the nodes in the $\theta$-partial $\mathrm{R}^{+}$-tree, which are $\{B, F, G, 11,14\}$, are same as the MBRs that we select during filtering step. In fact, the construction of a $\theta$ partial $\mathbf{R}^{+}$-tree is identical to filtering process using $\mathbf{R}^{+}$-tree. And according to the type of $\theta$, we can describe any kind of spatial query process using $R^{+}$-tree. Construction of a $\theta$-partial $\mathrm{R}^{+}$-tree is therefore a generalized process of filtering step. A generalized algorithm for constructing $\theta$-partial $\mathbf{R}^{+}$-tree is shown by Algorithm 1 .

\section{Procedure Build $\theta$ Partial $\mathrm{R}^{+}$-tree $\left(R\right.$ : root node of $\mathrm{R}^{+}$-tree, $\theta$ : filtering condition $)$} $N_{P}(0) \leftarrow\{R\} ; \quad \quad / / N_{P}(i)$ is set of selected nodes in level $i / /$ for level $i$ from 0 to $d \quad / / d$ is height of $R / /$

$$
\begin{aligned}
& \quad N \leftarrow \bigcup_{n \in N_{p}(i-1)} \text { children }(n) ; / / N \text { is set of all sibling nodes on a level // } \\
& \qquad N_{P}(i) \leftarrow\{n \mid n \in N \text { and satisfies condition } \theta\} ; \quad / / \text { statement 1-1 // } \\
& \text { end For } \\
& \text { return } N_{P}(d) ; \quad \text { // return MBR of candidate objects // }
\end{aligned}
$$
end For

End Procedure

Algorithm 1 Build $\theta$-partial $\mathrm{R}^{+}$-tree.

We now explain how to use this algorithm to find connectivity in visual database in the next section.

\section{TWO QUERY PROCESSING METHODS FOR CONNECTIVITY}

In order to apply $\theta$-partial $\mathrm{R}^{+}$-tree algorithm mentioned in the previous section to connectivity search query, we define $\theta$-condition as follows;

$\theta: N=\left\{m_{a}, m_{b}\right\} \cup\{n \mid n \in \operatorname{sibling}(R, i)$ and for $\exists x \in N, n$ is connected with $x\} \quad$ - (eq. 1) where $m_{a}, m_{b}$ are respectively MBRs of query objects $A$, and $B$ on level $i$. 
This condition implies that the set of nodes satisfying $\theta$, includes MBRs of given query objects $A$ and $B$, and MBRs which are connected with $A$ and $B$ in direct or indirect way. In fact, the set of nodes, $N$ in $\theta$ condition is a connected component. It is obvious that if two given objects are connected, them their MBR on each level in $\mathrm{R}^{+}$-tree are also connected. Based on the definition of $\theta$-condition, we can write a brief algorithm of query processing for connectivity search as Algorithm 2.

procedure ConnectivitySearch ( $R: \mathrm{R}^{+}$-tree, $A, B:$ query objects )

// find connectivity between $A$ and $B$ //

define $\theta$ as $N$ in (eq. 1);

CandidateMBRSet $\leftarrow$ Build $\theta$ PartialR ${ }^{+}$-tree $(R, \theta)$;

FilteredObjects $\leftarrow$ all objects in the nodes of CandidateMBRSet;

Objects $\leftarrow$ Refinement(FilteredObjects, $A, B$ );

End Procedure

Algorithm 2 Query Processing Algorithm for Connectivity Search.

This algorithm consists of two steps; filtering step by Build $\theta$ PartialR ${ }^{+}$-tree and refinement step. However, the major part of this algorithm is obviously filtering step, since it dominantly determines the performance of the query processing. Refinement step fulfills the connect condition with filtered objects by existing algorithm or similar filtering algorithm, it takes a little processing time. It suffices to define $\theta$ condition as connectivity for this step and Build $\theta$ PartialR ${ }^{+}$-tree function performs the filtering.

\subsection{A Simple Method for Connectivity using $\theta$-Partial $\mathbf{R}^{+}$-tree}

In order to complete Build $\theta \mathrm{PartialR^{+ }}$-tree function, we have to precise how to find the set of nodes $N$, satisfying $\theta$-condition on each level in a $\theta$-partial $\mathrm{R}^{+}$-tree. In other words, we should find connected component on each level $i$, which corresponds to $N p(i)$ in Algorithm 1 . In the two subsequent sections, we will see how $N p(i)$ can be found in efficient way, that is, how to perform statement $1-1$ in Algorithm 1 , when $\theta$-condition is given as (eq.1). Algorithm 3 illustrates a simple method to perform this statement.

Procedure SearchConnectedComponent $I(M:$ set of MBRs, $A$ and $B:$ object, $i:$ level)

$/ / \mathrm{M}$ is the set of MBRs whose parents are connected //

$m_{a} \leftarrow \operatorname{MBR}(A, i), m_{b} \leftarrow \operatorname{MBR}(B, i) ; \quad / /$ find MBRs of $A$ and $B$ on level $i / /$

$M p \leftarrow\left\{m_{a}\right\} ; \quad / / M p:$ set of candidates //

while(there is no change on $M p$ )

$M p \leftarrow M p U\{m \mid m \in M-M p$ and $m$ is directly connected with $M p\} ;$

// statement 3-1 //

end while

if $m_{b} \notin M p$, then return Fail; $/ / m_{a}$ and $m_{b}$ are in different connected component // end procedure

Algorithm 3 Finding Connected Component on Level $i$ : Simple Method. 
For example, a set of line segments and its $\mathrm{R}^{+}$-tree are given as Figure 6 , and we will see how Algorithm 2 and 3 perform query processing for connectivity.

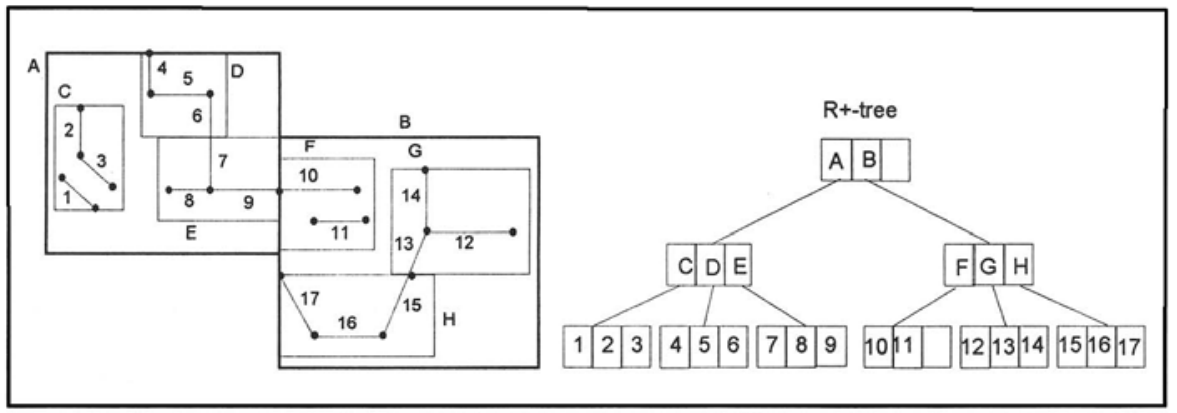

Figure 6 Line Segments and it's $\mathrm{R}^{+}$-tree.

Suppose that a connectivity query is given as 'Is line 14 connected with line 16 ?'. In SearchConnectedComponent 1 algorithm, $M p$ is $\{B\}$ on root level, and becomes $\{H, G\}$ on the next level, since $G$ is the MBR containing line 14, and $H$ is the only MBR which is connected with $G$. Thus, refinement step only has to check lines in $H$ and $G$. In this case, the number of disk accesses is at most four times, that is, one disk access for root node, node $B$, node $G$, node $H$, respectively. However, this filtering step requires slightly more CPU time. Figure 7 demonstrates detail procedure of the filtering step, that is Build $\theta$ Partial $R^{+}$-tree.

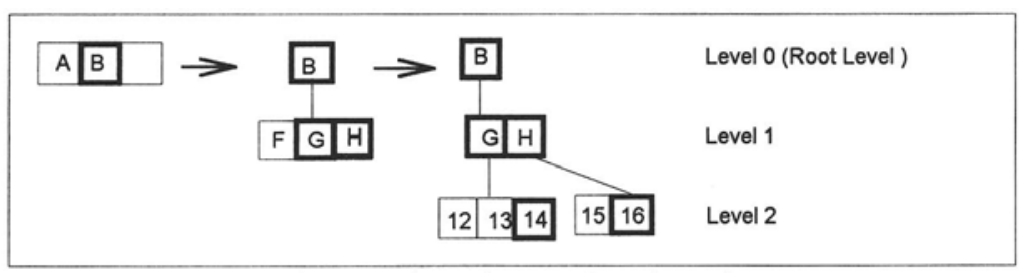

Figure 7 the procedure of processing given above query.

Another query example is 'Is line 12 connected with line 1 ?'. Then algorithm SearchConnectedComponent 1 finds MBRs of line 12 and line 1 on root level. That is, $\{A, B\}$ is taken on root level as connected component which is $M p$ in Algorithm 3. These nodes practically consist of nodes $\theta$-partial $\mathrm{R}^{+}$-tree on root level. On the next level, the connected component $M p$ containing the MBR of line 12 , becomes $\{H, G\}$, which does not include the MBR of line 1. It means that line 12 and 1 are not connected. It is therefore not necessary to continue any process in the algorithm, and we can get its answer that two line objects 12 and 1 are not connected. In this case, the number of disk accesses is 3 ; one for root node, one for node $B$ and one for node $C$. It implies that we can economize a lot of disk accesses and CPU time, when the query objects are disconnected. In this case, the height of $\theta$-partial $R^{+}$-tree may differs from the original $\mathrm{R}^{+}$-tree. In general, it can occur when the query objects are disconnected. That is, it becomes unnecessary to continue to buid $\theta$-partial $R^{+}$-tree, if it is proved that the MBRs of the objects are not connected. 
However, Algorithm 3 has room for improvement. In this algorithm, concrete processing method of statement $3-1$ is not described. The simplest method we can implement is to load all child nodes on main memory, whose parent nodes belong to the connected component, and process them. However, it significantly degrades the performance of this algorithm due to unnecessary disk accesses and computation. We will see an improved algorithm which avoids from such a problem in the next section.

\subsection{An Improved Method}

Let us consider an example illustrated by Figure 8 . Suppose that we have to find the connectivity between $a$ and $e$, which are MBRs on leaf level of query objects. Algorithm 3, SearchConnectedComponent $I$, needs no less than 26 comparisons, that is, 8 comparisons to extend the connected component, $M p$ from $\{e\}$ to $\{e, d\}$, and 7 for $M p=\{e, d, b\}$, and so on. However we can considerably reduce the number of comparisons and disk accesses by our improved method.

When we carefully look at the placements of MBRs in Figure 8. we can find that is

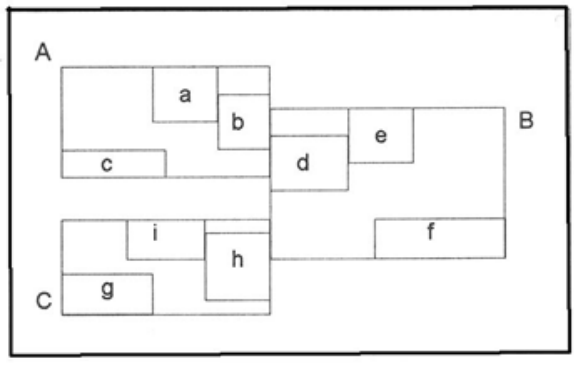
unnecessary to examine $C$ and its child nodes. It is because no child node of $B$ is connected with $C$. Therefore, we can remove unnecessary comparisons and corresponding disk accesses by using the following property;

Figure 8 Removing Unnecessary Disk Accesses and Comparisons.

\section{Property 1}

Let $M$ and $M^{\prime}$ be two different nodes on a same level in $R^{+}$-tree. If there exists no child node of $M$ which is connected with $M^{\prime}$, then there is no couple of child nodes $\left(m, m^{\prime}\right)$ which are connected, where $m$ and $m^{\prime}$ are in $M$ and $M^{\prime}$ respectively.

In Figure 8, we can see that any child node of $B$ is not connected with $C$ and consequently with any child node of $C$, according to the property. It implies that we can avoid disk access for node $C$ and comparisons with child nodes of $C$. We will analyze the improvement of performance in section 5 .

Algorithm 4 describes the improved procedure by using the property mentioned above. Before loading a node, this algorithm checks if it fulfills the property. The major differences between Algorithm 3 and 4 are that we inserted condition for property 1 and we explicitly specified when a node is to be loaded. This algorithm allows to load only the nodes which are relevant. For example, if we find the connectivity of $e$ and $a$ on leaf level in Figure 8, Algorithm 4 will be executed as follows; 
Initially, the input parameters of Algorithm 4 are $P=\{A, B, C\}$, and $A=e, B=a$.

Then the following processes of execution are;

step 1. Load $B$ node and set $M, M p$ as $\{d, e, f\}$ and $\{e, d\}$ respectively.

step 2. Check $C$ if it fulfills property 1 . fails.

step 3. Check $A$ if it fulfills property 1 , succeeds.

step 3-1. Load $A$, and find connected node with $M p$ among child nodes of $B$.

step 3-2. Update $M p$ as $M p \cup\{b, a\}$.

step 4. check if $a$ is in $M p$. Succeeds and terminate algorithm.

In this case, we see that $C$ is never loaded and its child nodes are not compared, whereas an additional disk access and computation for its child nodes are required by Algorithm 3. We will compare the performances of these algorithms in section 5 .

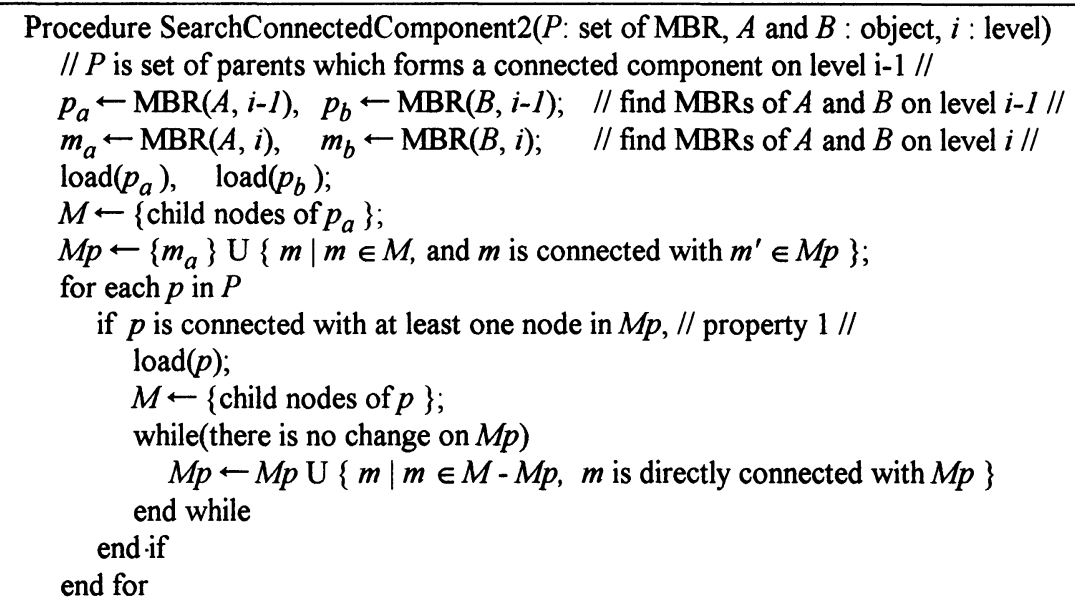

if $m_{b} \notin M p$, then return Fail; // $m_{a}$ and $m_{b}$ are in different connected component // end procedure

Algorithm 4 Improved Method for Finding Connected Component on Level $i$.

\section{PERFORMANCE}

In order to analyze the performance of query processing for connectivity using $\theta$-partial $\mathrm{R}^{+}$tree, we must consider three measures; filtering rate, number of disk accesses and CPU time. It is obvious that reducing the number of disk accesses is crucial in spatial query processing. The results of our experiments have shown that we can not ignore the CPU time needed for query processing of connectivity as well as disk $\mathrm{I} / \mathrm{O}$ time. The filtering rate, which can be defined as the rate of candidate objects over total objects, tells about how many unnecessary objects are removed by filtering process. Small filtering rate implies that the number of objects which are 0 be verified on refinement step is small. The more objects are removed, the less becomes the filtering rate. 
These three measures are, however correlated. For example, as filtering rate increase, the number of candidate objects increases and the amount of CPU time for refinement step may increase at the same time.

We simulated the performance of the two methods Algorithm 3 and 4 proposed in section 4, with 10000 segment objects, which were clustered into 100 regions, and $\mathrm{R}^{+}$-tree built from the objects. In reality, the distribution of objects is clustered rather than uniform [15][16]. We varied the relative size of objects over the size of total area from 0.0005 to 0.002 . In order to find out connectivity between two objects, we also generated 100 couples of query objects. The query objects were also distributed around clusters. The simulation was performed on Sparc workstation under Unix system. We observed CPU time, number of disk accesses and filtering rate.

Table 1 Filtering Rate

\begin{tabular}{|l|l|l|l|l|}
\hline Relative size & 0.0005 & 0.001 & 0.0015 & 0.002 \\
\hline Average number of $\mathrm{R}^{+}$-tree leaf nodes & 424 & 433 & 465 & 467 \\
\hline $\begin{array}{l}\text { Average number of leaf nods in } \theta \text {-partial } \mathrm{R}^{+} \text {- } \\
\text { tree }\end{array}$ & 47 & 212 & 244 & 366 \\
\hline Average filtering rate & 0.110 & 0.313 & 0.524 & 0.784 \\
\hline $\begin{array}{c}\text { Average number of internal nodes referenced } \\
\text { in } \theta \text {-partial } \mathrm{R}^{+} \text {-tree }\end{array}$ & 15.5 & 19.5 & 21.0 & 19.5 \\
\hline Average probability(filtering rate=0) & 0.825 & 0.685 & 0.475 & 0.215 \\
\hline
\end{tabular}

Table 1 shows the filtering rates of the methods proposed in the previous section, depending on the different relative sizes. When the relative size is large, the probability that two objects are connected is strong, and the number of objects in a connected component is also large. And it should be also noted that while their checking processes of $\theta$ condition in the two methods are different as explained in the previous section, the numbers of filtered nodes are identical. In table 1 , we can observe that the number of leaf nodes in $\mathrm{R}^{+}$-tree slightly differ depending on relative size. It is due to the handling policy of overlapping MBR in $\mathrm{R}^{+}$-tree construction algorithm.

We find that the filtering efficiency is excellent, when the relative size is very small $(=0.0005)$. Even though we count the CPU time and the numbers of disk accesses during filtering phase, which are eventually identical to the numbers of references on internal nodes in $\mathrm{R}^{+}$-tree, listed on fourth row, the overhead of filtering phases is not comparable with the benefits of our methods, which are reduction of total CPU time and disk accesses required.

But as the relative size grows, the filtering efficiency becomes worse. It is because our checking process is to find out connected components and when the relative size increase, the number of nodes in a connected component also increase. When the relative size is 0.0005 , the filtering rate is about 0.1 . It implies that the number of objects which are to be verified for refinement step, reduces by $1 / 10$. When we consider the CPU time to process refinement whose complexity is $O\left(n^{2}\right)$, we gain CPU cost by 100 times.

The last row in Table 1 means the probability that the filtering process detects that query objects are not connected and stops processing by returning NotConnected. In this case, no more disk accesses or CPU time become useless and the query processing performance is improved by considerable amount. 


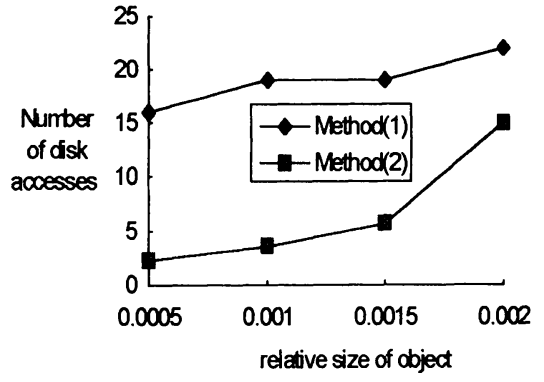

Figure 9 Number of Disk Accesses.

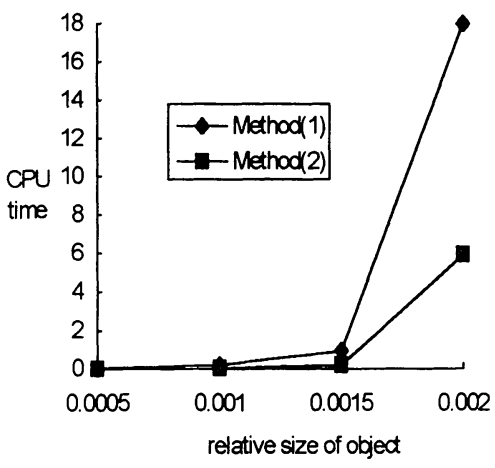

Figure 10 CPU time.

We compared the two methods proposed in section 4, by means of number of disk accesses and CPU time needed by filtering. Figure 9 shows the numbers of disk accesses of the methods depending on relative size. Algorithm 3 requires more disk accesses than Algorithm 4, as we already mentioned. However, the difference between the methods decreases, as increases the relative size.

Figure 10 shows CPU time for the two methods. We find a difference between the methods. For small objects, the performances of the methods slightly differ. But for large objects, we observe a great difference between the two methods.

\section{CONCLUSION}

To processing topological query in visual databases is in general very complicated and expensive. In this paper, we have proposed several spatial query processing methods for connectivity, using geometric data and $\mathrm{R}^{+}$-tree tree as the underlying indexing method, without any topological data. Our approach was inspired by filtering and refinement spatial query processing strategy. The goal is to minimize the number of disk accesses and CPU time for query processing, and on the same time to speed up the filtering process. To achieve these goals, we propose

i. A general spatial query processing method for filtering using $\theta$-partial $\mathbf{R}^{+}$-tree. We can employ this procedure for any kind of spatial query, by specifying $\theta$ condition and its checking method.

ii. Two checking methods when $\theta$ condition is connectivity. These methods speed up the filtering process by minimizing the number of disk accesses and CPU time.

We compared the performances of the proposed methods by simulation. The results of simulation showed that the second method gives better performance than the first. When the size of objects is small, the second method is excellent. And both of the methods considerably reduce the number of objects to be verified during refinement step. 
The connectivity query is a fundamental one in visual databases. Other types of queries can be derived from it. For example, the shortest path search query is an application of connectivity query. Further work could focus on the topics such like upstream, downstream, and shortest path query processing method based on connectivity. These are intensively required in many real applications of visual database systems.

Another focus of our researches includes the generalization of $\theta$-partial $\mathbf{R}^{+}$-tree to other types of query which requires complex and expensive processing. Also parallelization of $\theta-$ partial $\mathrm{R}^{+}$-tree with use of parallel R-trees [12] could be one of research focus.

\section{Acknowledgement}

I would like to thank $\mathrm{T}$. Sellis for giving me $\mathrm{R}^{+}$-tree source program.

\section{REFERENCES}

[1] Laurini,R. and Thompson,D. (1992) Fundamentals of Spatial Information Systems. $A$ cademic press, 470 pages.

[2] Guenther,O. and Buchmann,A. (1990) Research Issues in Spatial Databases. SIGMOD Record. Vol.19 No.4, pp.61-68.

[3] Agrawal,R. and Jagadish,H.V. (1994) Algorithms for Searching Massive Graphs. IEEE Trans. Knowledge and Data Engineering Vol.6 No.2, pp.225-238.

[4] Jagadish,H.V. (1990) On Indexing Line Segments. Proc. I6th VLDB Conf. , pp.614-625.

[5] Aho,A.V., Hopcroft,J.E. and Ullman,J.D. (1983) The Design and Analysis of Computer Algorithms. Addison-Wesley, 680 pages.

[6] Ioannidis,Y.E and Ramakrishnan,R. (1987) Efficient Transitive Closure Algorithms. Proc. 14th VLDB , pp.507-518.

[7] Brinkhoff,T., Kriegel,H.P. and Seeger,B. (1993) Efficient Processing of Spatial Joins Using R-trees. Proc. SIGMOD'93, pp.237-246.

[8] Orenstein,J. (1986) Spatial Query Processing in an Object-Oriented Database System. Proc. SIGMOD'86, pp.326-333.

[9] Sellis, T., Roussopoulos,N. and Faloutsos,C. (1987) The R+-tree: A Dynamic Index for Multidimensional Objects. Proc. VLDB, pp. 507-518.

[10] Guttman,A. (1984) R-trees: A Dynamic Index Structure for Spatial Searching. Proc. SIGMOD'84, pp.47-57.

[11] Beckmann,N., Kriegel,H.P., Schneider,R. and Seeger.B. (1990) The R*-tree: An Efficient and Robust Access method for Points and Rectangles. Proc. SIGMOD'90, pp.322-331.

[12] Kamel,I. and Faloutsos,C. (1992) Parallel R-tree. Proc. SIGMOD'92, pp.195-204.

[13] Graefe,G. (1993) Query Evaluation Techniques for Large Databases. ACM Computing Surveys, Vol.25 No2, pp.73-170.

[14] Lu,W. and Han,J. (1992) Distance-Associated Join Indices for Spatial Range Search. Proc. ICDE'92, pp.284-292.

[15] Hoel,E.G. and Samet,H. (1992) A Qualitive Comparison Study of Data Structures for Large Line Segment Databases. Proc. SIGMOD'92, pp.205-214.

[16] Faloutsos,C. and Kamel,I. (1994) Beyond Uniformity and Independence : Analysis of Rtree Using the Concept of Fractal Demension. Proc. PODS'94, pp.4-13. 


\section{BIOGRAPHY}

Myeong-A Kang received the B.S. degree in computer science from Pusan National University, South Korea, in 1994. She is currently a graduate student in department of computer science at Pusan National University.

Ki-Joune Li received the B.S. and M.S. degrees in computer science from Seoul National University, South Korea, and Ph.D from INSA, France, in 1992. He is currently in department of computer science at Pusan National University, South Korea. His current research interests include spatial information systems, hypermedia and object-oriented software engineering . 